\title{
A key to the females of Japanese tabanid flies with a checklist of all species and subspecies (Diptera, Tabanidae)
}

\author{
Hirofumi Hayakawa * \\ Upland Farming Division, Hokkaido National Agricultural Experiment Station, \\ Memuro-cho, Hokkaido 082, Japan
}

(Received: May 26, 1984)

Key words: Tabanidae, synonym, key, checklist, Japan.

Abstract: Key to the subfamilies, genera, species and subspecies of the female of Japanese tabanid flies is presented. One hundred and three species and subspecies are accepted as valid excluding two new synonyms.

The latest monograph of Tabanidae in Japan was published in 1969 by Murdoch and Takahasi. Since then, 39 new species and subspecies have been added to the fauna in Japan (Watanabe and Takahasi, 1971; etc.). Recently the author examined the type specimens in the collection of Dr. $\mathrm{H}$. Takahasi's (Hayakawa, 1984), and find that the following two species are new synonyms: Hybomitra akiyamai Murdoch and Takahasi, $1969=H y$. hirticeps (Loew, 1858). Tabanus tanbaensis Murdoch and Takahasi, 1969= T. administrans Schiner, 1868. As a result, 9 genera, 94 species and 9 subspecies should be accepted as valid in the taxonomy of Japanese tabanid flies.

\section{Key to the subfamilies and genera}

1. Antennal flagellum with less than 5 annulations $\ldots \ldots \ldots \ldots \ldots \ldots \ldots \ldots \ldots \ldots \ldots \ldots \ldots \ldots \ldots$ Antennal flagellum with 8 annulations ........ Subfamily Pangoniinae... Stonemyia

2. Hind tibia with spurs $\ldots \ldots \ldots \ldots \ldots \ldots \ldots \ldots$ Subfamily Chrysopinae... 3 Hind tibia without $\operatorname{spurs} \ldots \ldots \ldots \ldots \ldots \ldots \ldots$ Subfamily Tabaninae... 5

3. First segment of antennal flagellum cylindrical or plate-like $\ldots \ldots \ldots \ldots \ldots \ldots \ldots$ First segment of antennal flagellum ring-like $\ldots \ldots \ldots \ldots \ldots \ldots \ldots$ Nagatomyia

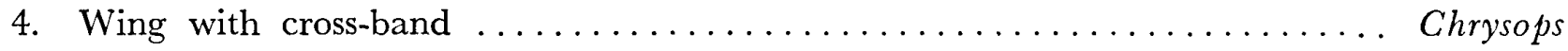

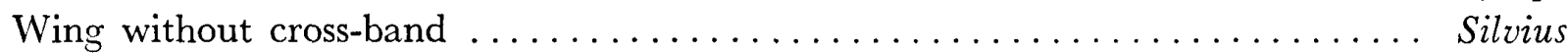

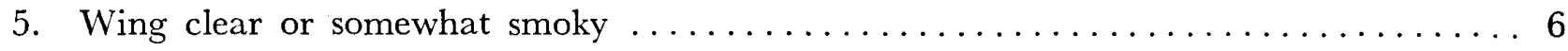
Wing brown with clear reticular designs $\ldots \ldots \ldots \ldots \ldots \ldots \ldots \ldots$ Haematopota

6. Dorsal process of antennal flagellum short or vestigial $\ldots \ldots \ldots \ldots \ldots \ldots \ldots \ldots .7$ Dorsal process of antennal flagellum very long, almost reaching the base of annulated

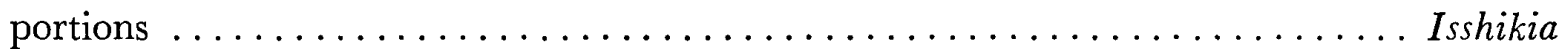

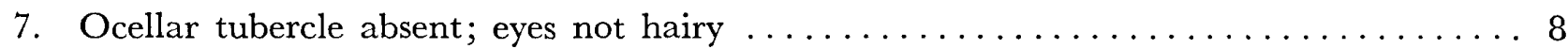
Ocellar tubercle present; eyes usually hairy $\ldots \ldots \ldots \ldots \ldots \ldots \ldots \ldots \ldots$ Hybomitra

8. Head normal; eyes blackish when dried, greenish in life; frontal callus large ......9 Head large; eyes ochre-yellow; frontal callus very small or absent ....... Atylotus

* 早川博文：農林水産省 北海道農業試験場 畑 作 部 （干082 北海道河西郡芽室町新生） 
9. Dorsal process of antennal flagellum distinctly projected $\ldots \ldots \ldots \ldots \ldots$ Tabanus Dorsal process of antennal flagellum slightly projected or vestigial ......... Hirosia

\section{Key to the species and subspecies (female)}

Genus Stonemyia Brennan, 1935

St. yezoensis and St. enokizonoi; but the latter is known only by male.

Genus Nagatomyia Murdoch and Takahasi, 1961

N. melanica only.

Genus Silvius Meigen, 1820

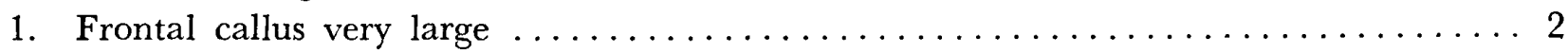

Frontal callus absent or vestigial $\ldots \ldots \ldots \ldots \ldots \ldots \ldots \ldots \ldots$ Si. formosensis

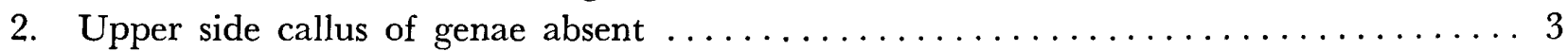

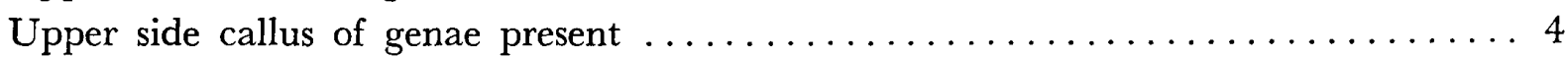

3. Wing clear; abdomen yellowish orange $\ldots \ldots \ldots \ldots \ldots \ldots \ldots \ldots$ Si. matsumurai

Wing brown; abdomen yellowish brown $\ldots \ldots \ldots \ldots \ldots \ldots \ldots \ldots$ Si. shirakii

4. Frontal callus very large, almost reaching eye-margins $\ldots \ldots \ldots \ldots \ldots$ Si. dorsalis

Frontal callus rather small, not reaching eye-margins $\ldots \ldots \ldots \ldots$ Si. oshimaensis

Genus Chrysops Meigen, 1800

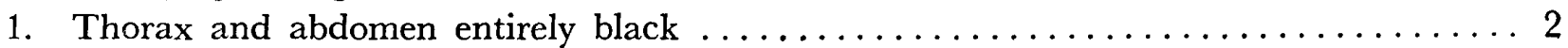

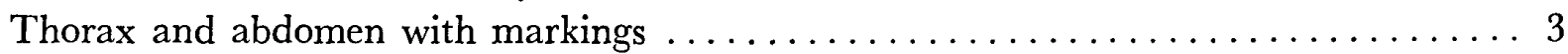

2. Wing with an apical marginal stripe $\ldots \ldots \ldots \ldots \ldots \ldots \ldots \ldots \ldots \ldots \ldots \ldots \ldots \ldots \ldots$. japonicus

Wing without an apical marginal stripe $\ldots \ldots \ldots \ldots \ldots \ldots \ldots$. yamaguchii

3. Abdominal terga 2 to 4 with 4 blackish vertical stripes $\ldots \ldots \ldots \ldots \ldots \ldots \ldots \ldots$

Abdominal terga 2 to 4 without 4 blackish vertical stripes $\ldots \ldots \ldots \ldots \ldots \ldots \ldots 10$

4. Sublateral stripes on abdominal tergum 2 broader at base $\ldots \ldots \ldots \ldots \ldots \ldots \ldots$

Sublateral stripes on abdominal tergum 2 narrower at base $\ldots \ldots \ldots \ldots \ldots \ldots 7$

5. Thorax and abdomen almost concolorous, yellow $\ldots \ldots \ldots \ldots \ldots \ldots \ldots \ldots \ldots$

Thorax and abdomen not concolorous; abdomen distinctly yellowish orange ......

C. vanderwulpi tanensis

6. Stripes on abdominal tergum 2 very broad; submedian and sublateral stripes almost connecting at base $\ldots \ldots \ldots \ldots \ldots \ldots \ldots \ldots \ldots \ldots \ldots \ldots \ldots \ldots \ldots \ldots$ vanderwulpi kitaensis

Stripes on abdominal tergum 2 rather narrow; submedian and sublateral stripes

separating at base $\ldots \ldots \ldots \ldots \ldots \ldots \ldots \ldots \ldots \ldots \ldots \ldots \ldots \ldots \ldots \ldots$ vanderwulpi saikaiensis

7. Thorax and abdomen yellow or yellowish orange, with distinct black stripes $\ldots . \ldots 8$

Thorax and abdomen yellowish brown, with somewhat indistinct black stripes ....

C. vanderwulpi sakishimaensis

8. Thorax and abdomen concolorous, yellow....................... 9

Thorax and abdomen not concolorous; abdomen distinctly yellowish orange .....

$\ldots \ldots \ldots \ldots \ldots \ldots \ldots \ldots \ldots \ldots \ldots \ldots \ldots \ldots \ldots \ldots \ldots \ldots \ldots$. vanderwulpi nanbuensis

9. Submedian stripes on abdominal tergum 2 bending sideways at the end .........

$\ldots \ldots \ldots \ldots \ldots \ldots \ldots \ldots \ldots \ldots \ldots \ldots \ldots \ldots \ldots \ldots \ldots$. vanderwulpi yamatoensis

Submedian stripes on abdominal tergum 2 bending innerways at the end

C. vanderwulpi stonei

10. Black markings on abdominal tergum 2 about reversed $V$-figure $\ldots \ldots \ldots \ldots \ldots 11$

Black markings on abdominal tergum 2 about spot or elongate-shaped $\ldots \ldots \ldots 12$

11. Markings on abdominal tergum 2 long, extending to sublateral parts ...... C. nigripes 
Markings on abdominal tergum 2 rather short, only extending to submedian parts

C. makerowi

12. Marking on abdominal tergum 2 large, reaching posterior margin $\ldots \ldots \ldots \ldots 13$

Markings on abdominal tergum 2 small, spot-like, separating from posterior margin

C. dissectus

13. Marking on abdominal tergum 2 longitudinal $\ldots \ldots \ldots \ldots \ldots \ldots \ldots$. suavis

Markings on abdominal tergum 2 horizontal .................. basalis

Genus Hybomitra Enderlein, 1922

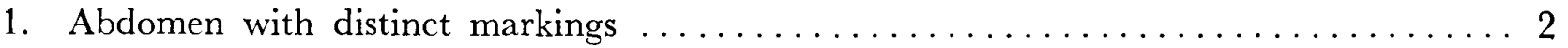

Abdomen without distinct markings, almost black ...........Hy. stigmoptera fujii

2. Markings on abdomen longitudinally rowed $\ldots \ldots \ldots \ldots \ldots \ldots \ldots \ldots \ldots \ldots$

Markings on abdomen horizontally striped, black and golden .......Hy. tarandina

3. Markings on abdomen white or greyish white $\ldots \ldots \ldots \ldots \ldots \ldots \ldots \ldots \ldots \ldots$

Markings on abdomen yellowish orange or reddish brown $\ldots \ldots \ldots \ldots \ldots \ldots \ldots$

4. Sublateral markings on abdomen round or oblique-rectangular $\ldots \ldots \ldots \ldots \ldots$

Sublateral markings on abdomen about triangular $\ldots \ldots \ldots \ldots \ldots \ldots \ldots \ldots \ldots \ldots \ldots \ldots$

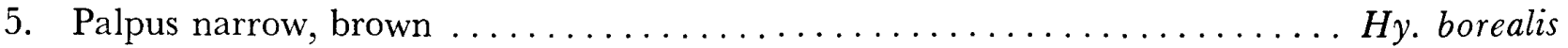

Palpus broad, light yellow $\ldots \ldots \ldots \ldots \ldots \ldots \ldots \ldots \ldots \ldots \ldots \ldots \ldots \ldots$ tsushimaensis

6. Subcallus nude, black ........................ Hy. hirticeps

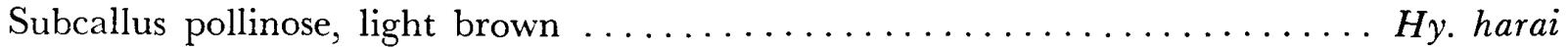

7. Sublateral markings on abdomen large; markings on terga 1 to 3 especially large,

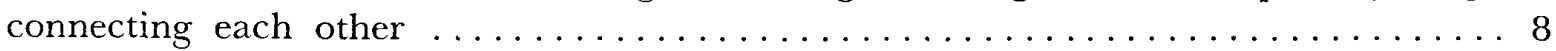

Sublateral markings on abdomen rather small; markings on terga 1 to 3 not especially

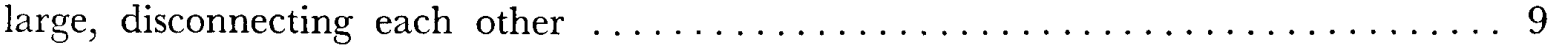

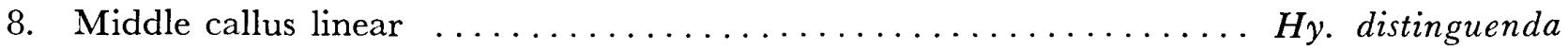

Middle callus fusiform $\ldots \ldots \ldots \ldots \ldots \ldots \ldots \ldots \ldots \ldots \ldots \ldots \ldots \ldots \ldots \ldots$ brevis

9. Sublateral markings on abdomen about the same size on each segment $\ldots \ldots \ldots \ldots$

Sublateral markings on abdomen especially large in tergum $2 \ldots \ldots \ldots \ldots$. . . jersey

10. Middle callus short, not exceeding half of frons $\ldots \ldots \ldots \ldots \ldots \ldots \ldots \ldots \ldots \ldots$

Middle callus long, exceeding half of frons $\ldots \ldots \ldots \ldots \ldots \ldots \ldots \ldots H y$. takahasii

11. Middle callus separating from basal callus $\ldots \ldots \ldots \ldots \ldots \ldots \ldots \ldots \ldots \ldots \ldots$

Middle callus connecting to basal callus ................Hy. ussuriensis

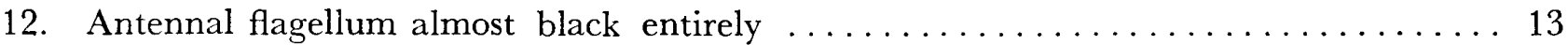

Antennal flagellum black only at style $\ldots \ldots \ldots \ldots \ldots \ldots \ldots \ldots \ldots$. ishiharai

13. Sublateral markings on abdomen distinct, orange $\ldots \ldots \ldots \ldots \ldots \ldots \ldots$. montana

Sublateral markings on abdomen indistinct, orange brown $\ldots \ldots \ldots \ldots \ldots \ldots H y$. olsoi

Genus Atylotus Osten Sacken, 1876

1. Frontal callus absent or vestigial; frons rather broad $\ldots \ldots \ldots \ldots \ldots \ldots \ldots \ldots$

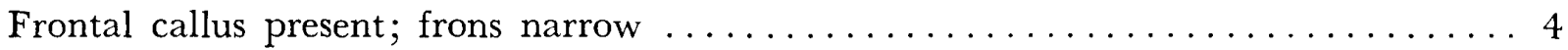

2. Median stripe on abdomen indistinct, brownish $\ldots \ldots \ldots \ldots \ldots \ldots \ldots \ldots \ldots \ldots$

Median stripe on abdomen distinct, golden $\ldots \ldots \ldots \ldots \ldots \ldots \ldots \ldots \ldots$. suzukii

3. Femur orange or yellowish orange $\ldots \ldots \ldots \ldots \ldots \ldots \ldots \ldots \ldots \ldots \ldots \ldots \ldots \ldots \ldots \ldots$ angusticornis

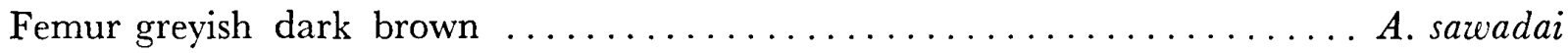

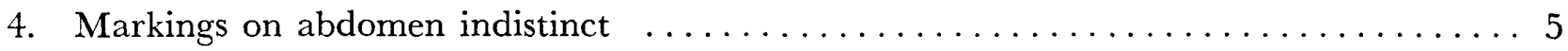

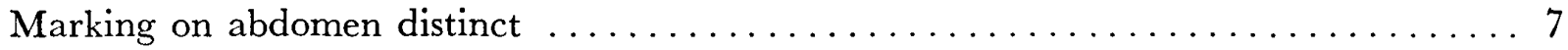

5. Abdomen yellowish brown; middle callus present $\ldots \ldots \ldots \ldots \ldots \ldots \ldots \ldots \ldots$ 
Abdomen greyish black with yellowish tint; middle callus absent ... A. aethereus sibricus

6. Appendix on wing vein $\mathrm{R}_{4}$ long

A. kakeromaensis

Appendix on wing vein $R_{4}$ very short $\ldots \ldots \ldots \ldots \ldots \ldots \ldots$ takaraensis

7. Median stripe on abdomen yellowish grey or black, darker than other parts ......8 Median stripe on abdomen yellow, brighter than other parts ..........A.keegani

8. Median stripe on abdomen as wide as on each tergum ............... Median stripe on abdomen narrowest on tergum $2 \ldots \ldots \ldots \ldots$....................

9. Lateral or sublateral markings on abdomen rather small, present on terga 1 to $6 \ldots 10$ Lateral markings on abdomen large, present on terga 1 to $3 \ldots \ldots$. . . . . . .

10. Sublateral markings on abdomen rather indistinct, yellow or greyish yellow

Sublateral markings distinct, creamy white

A. bivittateinus A. ozensis Genus Hirosia Hayakawa, 1983

1. Scutellum white or yellowish white, not concolorous with scutum ........... Scutellum yellow or blackish, concolorous with scutum ..............

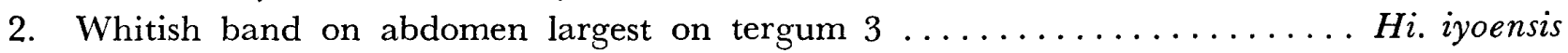
Whitish or yellowish white band on abdomen largest on tergum $2 \ldots$.... Hi. humilis

3. Thorax and abdomen almost brown to black ................... Thorax and abdomen entirely yellow .................Hi. sapporoensis

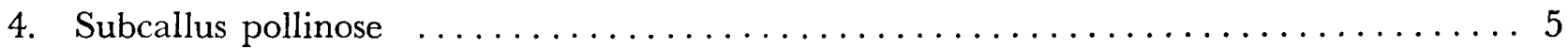
Subcallus nude .......................... amamiensis

5. Tibia dark brown to black . . . . . . . . . . . . . . . . . . . . . 6 Tibia white to greyish white $\ldots \ldots \ldots \ldots \ldots \ldots \ldots \ldots \ldots \ldots$ hyugaensis

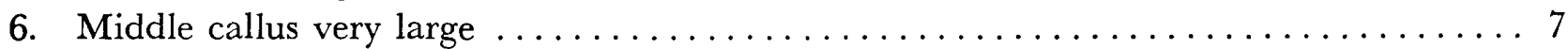

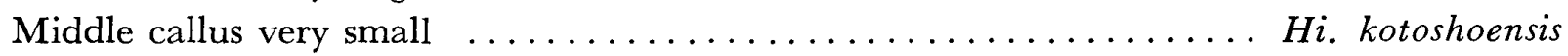

7. Basal callus about rectangular $\ldots \ldots \ldots \ldots \ldots \ldots \ldots \ldots \ldots \ldots \ldots \ldots \ldots \ldots \ldots \ldots \ldots \ldots$ Basal callus about round or semi-circular .................. Hi. otsurui

8. Middle callus connecting broadly to basal callus ................ Hi. daishojii Middle callus connecting linearly to basal callus ............... Hi. masamitsui

Genus Tabanus Linné, 1758

1. Wing vein $\mathrm{R}_{5}$ and $\mathrm{M}_{1}$ closed or slightly open $\ldots \ldots \ldots \ldots$

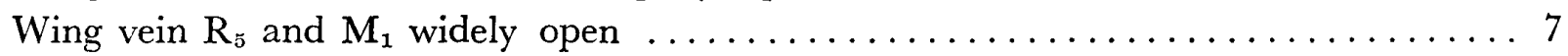

2. Median and sublateral markings on abdomen present $\ldots \ldots \ldots \ldots$ Median and sublateral markings on abdomen absent............ T. yoneyamai

3. Sublateral markings on abdomen present on terga 1 to $6 \ldots \ldots \ldots$ Sublateral markings on abdomen present only on terga 2 and $3 \ldots \ldots$ trigeminus

4. Antennal flagellum brown to orange yellow; abdomen olive with light olive markings ... 5 Antennal flagellum almost black; abdomen black with whitish markings ..........

T. takasagoensis

5. Sublateral markings on abdomen elongate-triangular $\ldots \ldots \ldots \ldots$

Sublateral markings on abdomen oblique-rectangular .......... T. pallidiventris

6. Middle callus rather short ........................ T. nipponicus Middle callus rather long, extending half of frons .............T. taiwanus

7. Middle callus spindle-shaped, slightly separating from middle callus ..........8 Middle callus not spindle-shaped, connecting to basal callus . . . . . . . . . . . 9

8. Lateral markings on abdomen present on tergum 2, light yellowish brown ..... 
T. fulvimedioides

Lateral markings on abdomen absent; abdomen light yellowish brown basaly .... T. fulvilineus

9. Middle callus clearly distinguishable from basal callus $\ldots \ldots \ldots \ldots \ldots \ldots \ldots \ldots \ldots 10$

Middle callus undistinguishable from basal callus $\ldots \ldots \ldots \ldots \ldots \ldots \ldots \ldots \ldots$

10. Median markings on abdomen about triangular $\ldots \ldots \ldots \ldots \ldots \ldots \ldots \ldots \ldots \ldots 11$

Median markings on abdomen about rectangular ............ T. ryukyuensis

11. Middle callus about the same size as basal callus . . . . . . . . . . . . . . . 12

Middle callus much smaller than basal callus ................ T. toshiokai

12. Sublateral markings on abdomen oblique-elongate or round $\ldots \ldots \ldots \ldots \ldots \ldots \ldots$

Sublateral markings on abdomen indistinct $\ldots \ldots \ldots \ldots \ldots \ldots \ldots \ldots$. monomiensis

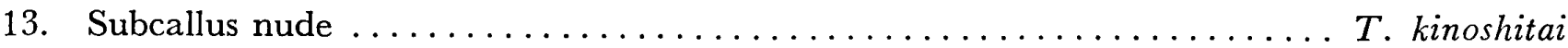

Subcallus pollinose $\ldots \ldots \ldots \ldots \ldots \ldots \ldots \ldots \ldots \ldots \ldots \ldots \ldots \ldots \ldots$ matsumotoensis

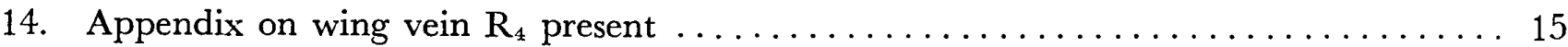

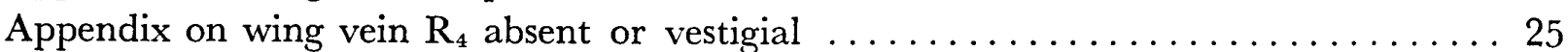

15. Middle callus very long, exceeding half of frons $\ldots \ldots \ldots \ldots \ldots \ldots \ldots \ldots \ldots$

Middle callus short, not exceeding half of frons $\ldots \ldots \ldots \ldots \ldots \ldots \ldots \ldots$. maedai

16. Sublateral markings on abdomen absent $\ldots \ldots \ldots \ldots \ldots \ldots \ldots \ldots \ldots \ldots \ldots \ldots$

Sublateral markings on abdomen present on terga 2 and 3, elongate-triangular ....

T. coquilletti

17. Subcallus light brown to yellowish brown, concolorous with frons $\ldots \ldots \ldots \ldots \ldots$

Subcallus white, not concolorous with frons $\ldots \ldots \ldots \ldots \ldots \ldots \ldots \ldots \ldots$ trigonus

18. Dorsal process of antennal flagellum well projected $\ldots \ldots \ldots \ldots \ldots \ldots \ldots \ldots \ldots$

Dorsal process of antennal flagellum not well projected $\ldots \ldots \ldots \ldots \ldots \ldots \ldots \ldots$

19. Abdomen reddish brown; median and posterior-marginal markings yellowish brown

T. matsuzawai

Abdomen greyish brown; median and posterior-marginal markings greyish white . .

20. Abdomen reddish brown to brown; median markings clear $\ldots \ldots \ldots \ldots \ldots \ldots \ldots \ldots$

Abdomen greyish brown; median markings indistinct ........... T. shikokuensis

21. Posterior-marginal markings on abdomen rather broad $\ldots \ldots \ldots \ldots \ldots \ldots \ldots \ldots \ldots 22$

Posterior-marginal markings on abdomen absent or very narrow $\ldots \ldots \ldots \ldots \ldots \ldots$

22. Middle callus connecting linearly to basal callus $\ldots \ldots \ldots \ldots \ldots \ldots \ldots \ldots \ldots \ldots$

Middle callus connecting broadly to basal callus .............. tokaraensis

23. Antennal flagellum reddish brown at base; wing hyaline $\ldots \ldots \ldots \ldots \ldots$. rufidens Antennal flagellum entirely black; wing light brown ........... . yaeyamaensis

24. Middle callus connecting linearly to basal callus; hairs on venter of thorax black ....

T. okinawanus

Middle callus connecting broadly to basal callus; hairs on venter of thorax yellowish

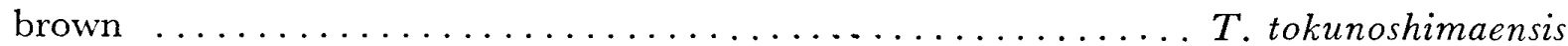

25. Dorsal process of antennal flagellum well projected $\ldots \ldots \ldots \ldots \ldots \ldots \ldots \ldots \ldots$

Dorsal process of antennal flagellum not well projected $\ldots \ldots \ldots \ldots \ldots \ldots \ldots \ldots$

26. Antennal flagellum yellowish orange at base .................. 27

Antennal flagellum entirely black $\ldots \ldots \ldots \ldots \ldots \ldots \ldots \ldots \ldots \ldots \ldots \ldots \ldots \ldots \ldots$ sapporoenus

27. Median triangular markings on abdomen absent or indistinct $\ldots \ldots \ldots \ldots \ldots \ldots$

Median triangular markings on abdomen clearly present $\ldots \ldots \ldots \ldots \ldots \ldots$ T. katoi 
28. Antennal flagellum entirely yellowish orange $\ldots \ldots \ldots \ldots \ldots \ldots \ldots \ldots$ chrysurus Antennal flagellum blackish brown at style $\ldots \ldots \ldots \ldots \ldots \ldots \ldots \ldots \ldots \ldots \ldots \ldots$ chrysurinus

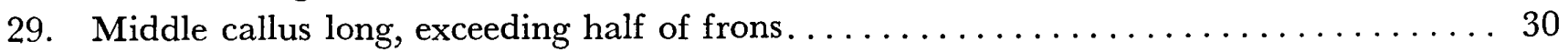

Middle callus short, not exceeding half of frons $\ldots \ldots \ldots \ldots \ldots \ldots \ldots \ldots \ldots \ldots$ sas

30. Abdominal terga 2 and 3 with median markings $\ldots \ldots \ldots \ldots \ldots \ldots \ldots \ldots \ldots \ldots$

Abdominal terga 2 and 3 without median markings $\ldots \ldots \ldots \ldots \ldots \ldots$. miyajima

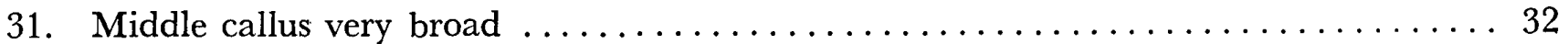

Middle callus linear; abdomen golden olive $\ldots \ldots \ldots \ldots \ldots \ldots \ldots \ldots \ldots \ldots \ldots \ldots \ldots$

32. Sublateral markings on abdomen present, elongate-triangular; wing hyaline .......

T. administrans

Sublateral markings on abdomen absent; wing distinctly brown ....T. yanbaruensis Genus Isshikia Shiraki, 1918

Abdomen dark brown to black; posterior-marginal markings greyish white ... I. yajimai Abdomen yellowish brown, especially at terga 1 to 3; posterior-marginal markings

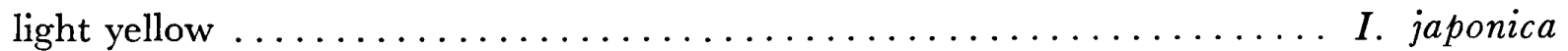

Genus Haematopota Meigen, 1803

1. Frons as broad as or narrower than the height $\ldots \ldots \ldots \ldots \ldots \ldots \ldots \ldots \ldots \ldots$ Frons much broader than the height $\ldots \ldots \ldots \ldots \ldots \ldots \ldots \ldots \ldots \ldots \ldots \ldots \ldots$ tamerlani

2. Basal segment of antennal flagellum very narrow, more than 2.5 times as long as the

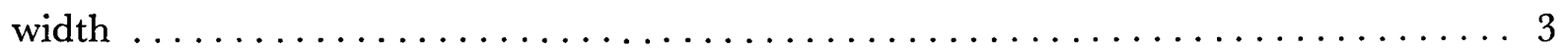

Basal segment of antennal flagellum very broad, about twice as long as the width ....

Ha. antennata

3. Antennal scape without a dorsal excision $\ldots \ldots \ldots \ldots \ldots \ldots \ldots \ldots \ldots \ldots \ldots$

Antennal scape with a dorsal excision at apex $\ldots \ldots \ldots \ldots \ldots \ldots \ldots \ldots$ Ha. tristis

4. Frontal callus not divided at upper point $\ldots \ldots \ldots \ldots \ldots \ldots \ldots \ldots \ldots \ldots \ldots \ldots \ldots \ldots \ldots$

Frontal callus divided at upper point $\ldots \ldots \ldots \ldots \ldots \ldots \ldots \ldots \ldots \ldots$. nagashimai

5. Frontal callus about chestnut-shaped; side spot on frons rather simple .........6 6 Frontal callus about rectangular; side spot on frons complicated ......Ha. shinonagai

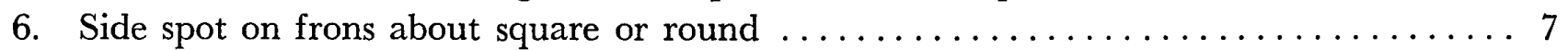
Side spot on frons elongate-oval with a little hollow on top ........Ha. kouzuensis

7. Side spot on frons about square $\ldots \ldots \ldots \ldots \ldots \ldots \ldots \ldots \ldots \ldots \ldots \ldots \ldots$

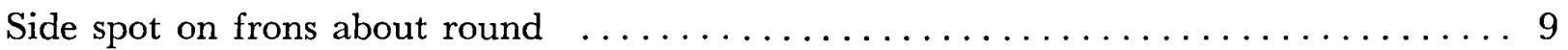

8. White spots present around appendix of wing vein $\mathrm{R}_{4} \ldots \ldots \ldots \ldots$ Ha. hakusanensis White spots absent around appendix of wing vein $\mathrm{R}_{4} \ldots \ldots \ldots \ldots$ Ha. toyamaensis

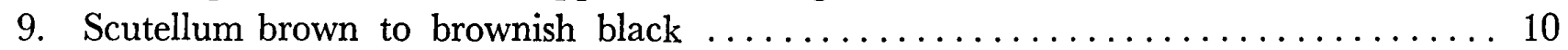
Scutellum greyish white $\ldots \ldots \ldots \ldots \ldots \ldots \ldots \ldots \ldots \ldots \ldots \ldots \ldots \ldots$. rufipennis

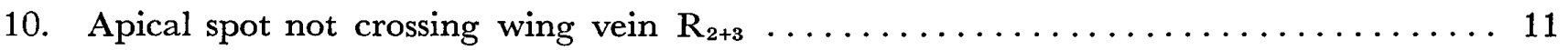
Apical spot crossing wing vein $R_{2+3} \ldots \ldots \ldots \ldots \ldots \ldots \ldots \ldots \ldots \ldots \ldots \ldots$ nasuensis

11. White spots on wing rather large $\ldots \ldots \ldots \ldots \ldots \ldots \ldots \ldots \ldots \ldots \ldots \ldots$ hikosanensis White spots on wing very small $\ldots \ldots \ldots \ldots \ldots \ldots \ldots \ldots \ldots \ldots \ldots \ldots \ldots$. surugaensis

Checklist of tabanid species and subspecies in Japan $\dagger$

1. Stonemyia yezoensis (Shiraki, 1918) マルガタアブ “Hokkaido, Honshu, Shikoku, Kyushu, Amami Islands"

2. St. enokizonoi (Ouchi, 1940) エノキゾノアブ "Yaku Island”

$\dagger$ Localities are shown in parenthesis. 
3. Nagatomyia melanica Murdoch and Takahasi, 1961 ムカシアブ "Honshu, Kyushu, Amami Islands"

4. Silvius dorsalis Coquillett, 1898 ヒメアブ "Hokkaido, Honshu, Kyushu"

5. Si. oshimaensis Hayakawa, Takahasi and Suzuki, 1982 オオシマヒメアブ “Amami Islands"

6. Si. matsumurai Kono and Takahasi, 1939 マッムラヒメアブ "Hokkaido, Honshu,

Shikoku"

7. Si. shirakii Philip and Mackerras, 1959 シラキヒメアブ "Nansei Islands"

8. Si. formosensis Ricardo, 1913 タイワンヒメアブ “Honshu, Shikoku, Nansei Islands"

9. Chrysops japonicus Wiedemann, 1828 クロメクラアブ “Hokkaido, Honshu, Shikoku, Kyushu"

10. C. yamaguchii Shimizu and Takahasi, 1975 ヤマグチメクラアブ “Honshu"

11. C. nigripes Zetterstedt, 1840 ヒメカラフトメクラアブ "Hokkaido"

12. C. makerowi Pleske, 1910 カラフトメクラアブ "Hokkaido"

13. C. dissectus Loew, 1858 マンシュウメクラアブ “Honshu, Kyushu”

14. C. suavis Loew, 1858 メクラアブ “Hokkaido, Honshu, Shikoku, Kyushu"

15. C. basalis Shiraki, 1918 キゴシメクラアブ “Hokkaido, Honshu”

16. C. vanderwulpi sakishimaensis Hayakawa, 1983 サキシマヨスジメクラアブ “Sakaishima Islands"

17. C. vanderwulpi nanbuensis Hayakawa, 1983 ホソヨスジメクラアブ "Honshu"

18. C. vanderwulpi yamatoensis Hayakawa, 1983 ヨスジメクラアブ "Hokkaido, Honshu, Shikoku, Kyushu"

19. C. vanderwulpi stonei Pechuman, 1977 オシマヨスジメクラアブ “Hokkaido"

20. C. vanderwulpi tanensis Hayakawa, 1983 タネヨスジメクラアブ “Tanegashima Island”

21. C. vanderwulpi kitaensis Hayakawa, 1983 キタヨスジメクラアブ “Hokkaido"

22. C. vanderwulpi saikaiensis Hayakawa, 1983 サイカイヨスジメクラアブ “Kyushu"

23. Hybomitra stigmoptera fujii Murdoch and Takahasi, 1969 ニッポンクロアブ “Honshu"

24. Hy. tarandina (Linné, 1761) カラフトアカアブ" “Hokkaido, Honshu"

25. Hy. hirticeps (Loew, 1858) マルヒゲアブ "Honshu"

Hy. akiyamai Murdoch and Takahasi, 1969, n. syn.

26. Hy. harai Hayakawa and Takahasi, 1976 ハラキボシアブ "Honshu"

27. Hy. brevis (Loew, 1858) ヒゲクロアブ "Honshu"

28. Hy. distinguenda (Verrall, 1909) キバラアブ "Hokkaido, Honshu”

29. Hy. jersey (Takahasi, 1950) ジャーシーアブ "Honshu”

30. Hy. takahasii Inaoka and Hayakawa, 1982 タカハシキボシアブ “Hokkaido"

31. Hy. ussuriensis (Olsoufiev, 1937) ウスリーキボシアブ “Hokkaido"

32. Hy. ishiharai (Takahasi, 1950) イシハラアブ "Honshu, Kyushu"

33. Hy. tsushimaensis Hayakawa, Yoneyama and Inaoka ツシマキボシアブ “Tsushima Islands"

34. Hy. borealis (Fabricius, 1981) コムラアブ "Hokkaido"

35. Hy. montana (Meigen, 1820) キボシアブ "Hokkaido, Honshu"

36. Hy. olsoi Takahasi, 1962 ホソヒゲキボシアブ "Hokkaido"

37. Atylotus suzukii Hayakawa, 1978 スズキキイロアブ "Shikoku, Kyushu”

38. A. angusticornis (Loew, 1858) オキナワキイロアブ "Ryukyu Islands"

39. A. sawadai Watanabe and Takahasi, 1971 サワダキイロアブ “Amami Islands”

40. A. keegani Murdoch and Takahasi, 1969 キーガンキイロアブ “Ryukyu Islands” 
41. A. aethereus sibricus (Olsoufiev, 1936) シベリアキイロアブ “Hokkaido"

42. A. kakeromaensis Hayakawa, Takahasi and Suzuki, 1982 カケロマキイロアブ “Amami Islands"

43. A. takaraensis Hayakawa and Takahasi, 1983 タカラキイロアブ "Tokara Islands"

44. A. horvathi (Szilady, 1926) ホルバートアブ "Hokkaido, Honshu, Shikoku, Kyushu"

45. A. hasegawai Hayakawa, 1978 ハセガワキイロアブ “Honshu”

46. A. bivittateinus Takahasi, 1962 フタスジアブ "Hokkaido, Honshu, Shikoku, Kyushu"

47. A. ozensis Hayakawa, 1983 オゼキイロアブ "Honshu"

48. Hirosia sapporoensis (Shiraki, 1918) キンイロアブ "Hokkaido, Honshu, Kyushu"

49. Hi. amamiensis (Hayakawa, Suzuki and Nagashima, 1981) アマミクロアブ “Amami Islands"

50. Hi. hyugaensis (Hayakawa, 1977) ヒュウガクロアブ "Honshu, Kyushu"

51. Hi. iyoensis (Shiraki, 1918) イヨシロオビアブ "Hokkaido, Honshu, Shikoku, Kyushu"

52. Hi. humilis (Coquillett, 1898) アオコアブ "Honshu"

53. Hi. kotoshoensis (Shiraki, 1918) チビアブ "Yaeyama Islands"

54. Hi. otsurui (Ogawa, 1960) オオツルアブ "Honshu"

55. Hi. daishojii (Murdoch and Takahasi, 1969) ダイショウジアブ “Honshu"

56. Hi. masamitsui (Hayakawa, 1976) マサミッアブ “Okinawa Island”

57. Tabanus chrysurus Shiraki, 1918 アカウシアブ “Hokkaido, Honshu, Shikoku, Kyushu”

58. T. chrysurinus (Enderlein, 1925) ニセアカウシアブ "Honshu, Shikoku, Kyushu"

59. T. sapporoenus Shiraki, 1918 アカアブ "Hokkaido, Honshu”

60. T. katoi Kono and Takahasi, 1940 カトウアカアブ “Hokkaido, Honshu"

61. T. yoneyamai Hayakawa, 1984 ヨネヤマアブ "Honshu"

62. T. trigeminus Coquillett, 1898 シロフアブ “Hokkaido, Honshu, Shikoku, Kyushu"

63. T. takasagoensis Shiraki, 1918 ギシロフアブ "Hokkaido, Honshu, Shikoku, Kyushu, Amami Islands"

64. T. pallidiventris Olsoufiev, 1937 マンシュウシロフアブ “Hokkaido, Honshu, Shikoku, Kyushu"

65. T. nipponicus Murdoch and Takahasi, 1969 ニッポンシロフアブ "Hokkaido, Honshu"

66. T. taiwanus Hayakawa and Takahasi, 1983 タイワンシロファブ “Honshu, Kyushu, Nansei Islands"

67. T. kinoshitai Kono and Takahasi, 1939 キノシタシロフアブ "Hokkaido, Honshu, Shikoku, Kyushu"

68. T. matsumotoensis Murdoch and Takahasi, 1961 マツモトアブ "Honshu, Shikoku"

69. T. monomiensis Takahasi, 1950 モノミクロバラアブ "Honshu, Kyushu"

70. T. toshiokai Murdoch and Takahasi, 1969 トシオカアブ “Shikoku, Kyushu, Amami Islands"

71. T. maedai Hayakawa, 1976 マエダアブ "Honshu"

72. T. trigonus Coquillett, 1898 ウシアブ “Hokkaido, Honshu, Shikoku, Kyushu"

73. T. shikokuensis Murdoch and Takahasi, 1961 シコクアブ “Shikoku"

74. T. inaensis Asakawa and Takahasi, 1975 イナウシアブ "Honshu"

75. T. coquilletti Shiraki, 1918 ハタケヤマアブ "Hokkaido, Honshu, Shikoku, Kyushu"

76. T. matsuzawai Hayakawa and Takahasi, 1983 マツザワアブ “Honshu, Shikoku, Kyushu"

77. T. rufidens (Bigot, 1887) ヤマトアブ "Hokkaido, Honshu, Shikoku, Kyushu"

78. T. yaeyamaensis Hayakawa and Hasegawa, 1981 ヤエヤマヤマトアブ "Yaeyama Islands"

79. T. okinawanus Shiraki, 1918 オキナワオオアブ “Okinawa Islands" 
80. T. tokunoshimaensis Hayakawa and Suzuki, 1984トクノシマオオアブ “Amami Islands"

81. T. tokaraensis Hayakawa and Suzuki, 1984 トカラヤマトアブ “Tokara Islands"

82. T. miyajima Ricardo, 1911 シロスネアブ “Honshu, Shikoku, Kyushu”

83. T. kanoi Murdoch and Takahasi, 1961 カノウアブ "Shikoku, Kyushu, Amami Islands"

84. T. ryukyuensis Murdoch and Takahasi, 1969 リュウキュウアブ "Nansei Islands"

85. T. yanbaruensis Hayakawa and Yoneyama, 1983 ヤンバルアブ "Okinawa Islands"

86. T. administrans Schiner, 1868 オカダアブ "Honshu, Kyushu"

T. tanbaensis Murdoch and Takahasi, 1969, n. syn.

87. T. sasai Watanabe and Takahasi, 1971 サッサアブ "Honshu"

88. T. fulvimedioides Shiraki, 1918 キスジアブ "Hokkaido, Honshu, Shikoku, Kyushu"

89. T. fulvilineus Hayakawa and Takahasi, 1961 ヒメキスジアブ “Honshu, Kyushu"

90. Isshikia japonica (Bigot, 1892) ヒゲナガサシアブ "Honshu, Shikoku, Kyushu"

91. I. yajimai Murdoch and Takahasi, 1961 ヤジマサシアブ "Honshu, Kyushu"

92. Haematopota tamerlani (Szilady, 1923) シベリアゴマフアブ “Hokkaido"

93. Ha. antennata (Shiraki, 1932) ヒゲブトゴマフアブ “Tsushima Islands”

94. Ha. tristis Bigot, 1981 ゴマフアブ “Hokkaido, Honshu”

95. Ha. kouzuensis (Takahasi, 1950) コウズゴマファブ "Honshu"

96. Ha. rufipennis Bigot, 1891 アカバゴマフアブ "Honshu, Shikoku, Kyushu”

97. Ha. toyamaensis Watanabe, Kamimura and Takahasi, 1976 トヤマゴマフアブ “Honshu”

98. Ha. nagashimai Hayakawa and Takahasi, 1976 ナガシマゴマフアブ "Honshu"

99. Ha. hakusanensis Tagashi, 1977 ハクサンゴマファブ “Honshu”"

100. Ha. shinonagai Hayakawa, 1977 シノナガゴマフアブ “Honshu”

101. Ha. hikosanensis Hayakawa and Takahasi, 1977 ヒコサンゴマフアブ "Shikoku, Kyushu”

102. Ha. surugaensis Hayakawa and Takahasi, 1977 スルガゴマフアブ “Honshu”

103. Ha. nasuensis Hayakawa and Moriyama, 1981 ナスゴマファブ "Honshu”

\section{REFERENCES}

Hayakawa, H. (1984): List of Tabanidae (Diptera) preserved at Dr. Hirosi Takahasi's, 1. Tabanid flies from Japan. Misc. Publ. Tohoku Natl. Agric. Exp. Stn., 4: 113-128.

Murdoch, W. P. and H. Takahasi (1969): The female Tabanidae of Japan, Korea and Manchuria. Mem. Entomol. Soc. Wash., 6: 230 pp.

Watanabe, M. and H. Takahasi (1971): Descriptions of two new species of horse flies from Japan (Diptera: Tabanidae). Kontyú, Tokyo, 39: $132-135$.

\section{日本産アブ科雌成虫の検索表と全種の目録}

Murdoch and Takahasi (1969) 以後に報告され た日本産アブ科の種を整理し，亜種を含めて 103 種が 産することを確認した. その中で, アキヤマアブ Hybomitra akiyamai Murdoch and Takahasi, 1969 はマルヒゲアブ Hy. hirticeps (Loew，1858) の，ま たタンバアブ Tabanus tanbaensis Murdoch and Takahasi, 1969 はオカダアブ T. administrans Schiner, 1968 の同物異名であることを明らかにした. 亜科, 属, 種 (雌)の検索表を作製し, チェックリスト を掲げた。 